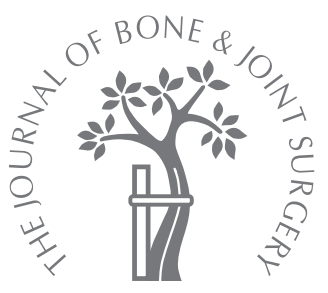

Y. Yonekura, H. Miyamoto, T. Shimazaki, Y. Ando, I. Noda, M. Mawatari, T. Hotokebuchi

From Saga University, Saga, Japan
- Y. Yonekura, MD,

Orthopaedic Surgeon

- T. Shimazaki, MD, PhD,

Orthopaedic Surgeon

M. Mawatari, MD, PhD,

Professor

- T. Hotokebuchi, MD, PhD

Department of Orthopaedic

Surgery

H. Miyamoto, PhD, Professor

Department of Pathology and

Microbiology

Faculty of Medicine, Saga

University, 5-1-1 Nabeshima,

Saga 849-8501, Japan.

Y. Ando, PhD, Research

Fellow

II. Noda, Research Fellow

Research Department

Japan Medical Materials

Corporation, 3-3-31 Miyahara,

Yodogawa-ku, Osaka, Japan.

Correspondence should be sent to $\mathrm{Dr}$ Y. Yonekura; e-mail: yoneyuta@acc.saga-u.ac.jp

(c)2011 British Editorial Society of Bone and Joint Surgery doi:10.1302/0301-620X.93B5. $25518 \$ 2.00$

$J$ Bone Joint Surg $[\mathrm{Br}]$

2011;93-B:644-9.

Received 9 July 2010; Accepted after revision 14 December 2010

\title{
Osteoconductivity of thermal-sprayed silver- containing hydroxyapatite coating in the rat tibia
}

\author{
A silver-containing hydroxyapatite (Ag-HA) coating has been developed using thermal \\ spraying technology. We evaluated the osteoconductivity of this coating on titanium (Ti) \\ implants in rat tibiae in relation to bacterial infection in joint replacement. \\ At 12 weeks, the mean affinity indices of bone formation of a Ti, an $\mathrm{HA}$, a 3\%Ag-HA and a \\ $50 \%$ Ag-HA coating were $97.3 \%, 84.9 \%, 81.0 \%$ and $40.5 \%$, respectively. The mean affinity \\ indices of bone contact of these four coatings were $18.8 \%, 83.7 \%, 77.2 \%$ and $40.5 \%$, \\ respectively. The indices of bone formation and bone contact around the implant of the \\ 3\%Ag-HA coating were similar to those of the HA coating, and no significant differences \\ were found between them (bone formation, $p=0.99$; bone contact, $p=0.957$ ). However, \\ inhibition of bone formation was observed with the $50 \% \mathrm{Ag}-\mathrm{HA}$ coating. \\ These results indicate that the $3 \% \mathrm{Ag}-\mathrm{HA}$ coating has low toxicity and good \\ osteoconductivity, and that the effect of silver toxicity on osteoconductivity depends on the \\ dose.
}

The rate of primary infection for joint replacements is between $0.86 \%$ and $2.52 \%$ according to the National Nosocomial Infections Surveillance System. ${ }^{1}$

Various antibacterial coatings such as vancomycin, ${ }^{2}$ gentamicin, ${ }^{3}$ a combination of gentamicin and hydroxyapatite (HA), ${ }^{4}$ carbonated $\mathrm{HA},{ }^{5}$ nitric oxide-releasing xerogel ${ }^{6}$ and silver (Ag)-containing $\mathrm{HA},{ }^{7}$ have been developed for use on the surface of implants. Devices such as heart valves, ${ }^{8}$ central venous catheters, ${ }^{9}$ aortic grafts, ${ }^{10}$ urethral catheters, ${ }^{11}$ burn wound dressings ${ }^{12}$ and megaprostheses, ${ }^{13}$ have been coated with Ag or Ag compounds because of their broad antibacterial spectrum, strong bactericidal activity and low toxicity. ${ }^{14,15}$

HA has good biocompatability and osteoconductivity and is used on the surface of implants to improve the strength of the attachment to bone. ${ }^{16-20}$ An antibacterial coating with HA containing silver oxide $\left(\mathrm{Ag}_{2} \mathrm{O}\right)$, which has the properties of both $\mathrm{Ag}$ and $\mathrm{HA}$, has been developed using thermal spraying. ${ }^{21}$ It has been reported that this coating has the properties of $\mathrm{HA}$, induces the release of $\mathrm{Ag}$ ions, has high antibacterial activity with inhibition of bacterial adhesion and has low cytotoxicity in vitro. ${ }^{21,22}$ It also demonstrates antibacterial activity against methicillinresistant Staphylococcus aureus (MRSA) in vivo ${ }^{23}$ as well as in vitro. ${ }^{22}$
However, an in vivo study on the osteoconductivity of such an Ag-HA coating has not been carried out. We have evaluated the osteoconductivity of this coating on titanium (Ti) implants in rat tibiae.

\section{Materials and Methods}

Coating and implants. The implants used were Ti wires $20 \mathrm{~mm}$ in length and $1 \mathrm{~mm}$ in diameter (HOMS, Nagano, Japan). The $\mathrm{Ag}_{2} \mathrm{O}$ powder (Kanto Chemical, Tokyo, Japan) was mixed with the HA powder (Japan Medical Materials, Osaka, Japan) in concentrations of $3 \%$ and $50 \%$ and shaken for five minutes in a plastic bag. The mix was then sprayed on to the Ti surface using the Flame Spraying System (Sulzer Metco Japan Ltd., Tokyo, Japan) which uses an acetylene torch. The temperature of the flame was about $2700^{\circ} \mathrm{C}$. We prepared four types of implant as follows: $\mathrm{Ti}$, HA coating on $\mathrm{Ti}$, $3 \% \mathrm{Ag}-\mathrm{HA}$ coating on $\mathrm{Ti}$ and $50 \% \mathrm{Ag}-\mathrm{HA}$ coating on Ti. They were packaged singly and sterilised using a JS-8500 gamma steriliser (MDS Nordion, Ontario, Canada). The implants were obtained from Japan Medical Materials. Previous studies in vivo and in vitro had indicated that the concentration of Ag in the coating was responsible for the antibacterial activity. ${ }^{22,23} \mathrm{An}$ amorphous structure of the Ag-HA coating was confirmed for concentrations $\leq 50 \% \mathrm{Ag}$. 


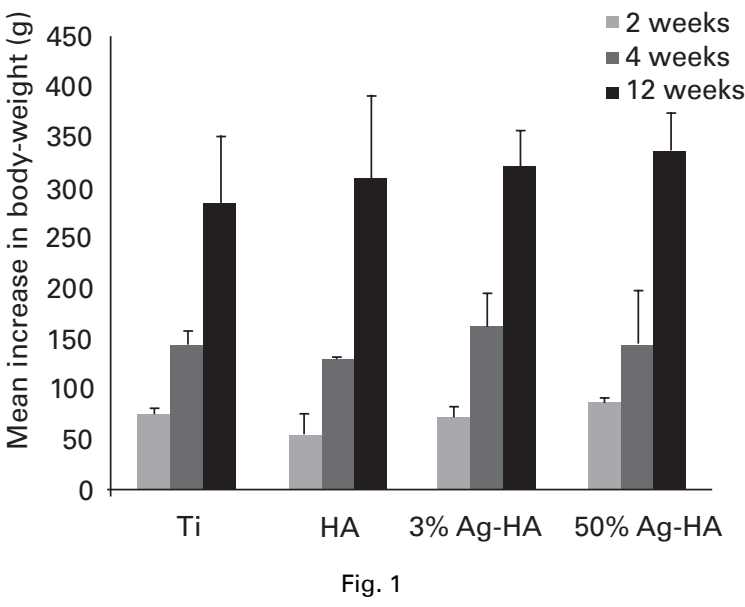

Bar charts showing the mean increase in body-weight at two, four and 12 weeks in the $\mathrm{Ti}$, hydroxyapatite (HA), $3 \% \mathrm{Ag}-\mathrm{HA}$ and $50 \% \mathrm{Ag}-\mathrm{HA}$ coating groups.

Operative technique. We used 38 10-week-old male Sprague-Dawley rats (Kyudo, Saga, Japan) with a mean weight of $364.6 \mathrm{~g}$ (335 to 430). The operation was carried out under general anaesthesia induced intra-peritoneally with pentobarbital (50 mg/kg body-weight). The right hind leg was shaved, cleaned with povidone-iodine and dried. A hole was drilled with an 18-gauge needle through the tibial tuberosity and the four different implants inserted into the medullary cavity of the tibiae. The rats were divided into four groups of three or four according to the implant inserted. They were housed individually with free access to food and water. After two, four and 12 weeks the bodyweight was measured. The abdomen was then opened under general anaesthesia and $5 \mathrm{ml}$ of blood was collected from the right common iliac vein. The tibia of the right hind leg was excised and the rats were killed. All the animal experiments were approved by the Animal Research Ethics Committee of Saga University and according to their recommendation that the number of experimental animals be reduced as much as possible, the sample was limited to three or four rats per group, which was the minimum number needed to calculate the mean and SD.

Radiological transmission imaging. At two, four and 12 weeks the radiographs were assessed for possible aseptic loosening, osteolysis and the formation of heterotopic bone, as described previously. ${ }^{13}$ The tibiae were analysed by radiological transmission imaging with microfocus $\mathrm{x}$-ray apparatus (SMX-225CT-SV2; Shimadzu, Kyoto, Japan).

Histological examination. The tibial specimens were fixed in $10 \%$ phosphate-buffered formaldehyde, dehydrated in graded alcohol solutions and embedded in polyester resin. Sections $300 \mu \mathrm{m}$ to $500 \mu \mathrm{m}$ thick were cut by a diamondbladed slicer (Maruto Co., Tokyo, Japan), ground to a thickness of $120 \mu \mathrm{m}$ to $150 \mu \mathrm{m}$ using a speed-track machine (Maruto Co.) and stained with toluidine blue. Osteoconductivity, bone formation and bone contact on the surface of the implant were evaluated by light microscopy. Affinity indices, describing the percentage of bone formation and bone contact, were calculated by analysis software (Image-Pro Plus version 4.0; Media Cybernetics, Silver Spring, Maryland). ${ }^{16,18,24}$ The bone formation index was calculated as the length of bone formation on the surface of the implant divided by the total length of this surface, multiplied by 100 . The bone contact index was calculated as the length of bone in direct contact with the surface of the implant divided by the total length of the implant, multiplied by 100 .

Serum analysis. The concentration of $\mathrm{Ag}$ in the serum was determined by inductively-coupled plasma-mass spectrometric analysis (Perkin Elmer, Waltham, Massachusetts).

Statistical analysis. This was performed with Excel and SPSS version 12.0J software (SPSS Inc., Chicago, Illinois). One-way analysis of variance was used to evaluate the body-weight, affinity index and serum analysis. Where this was significant, Tukey's HSD (honestly significant difference) was applied as a post-hoc test. A p-value $\leq 0.05$ was considered to be significant.

\section{Results}

Body-weight. The mean increase in body-weight at 12 weeks in the $\mathrm{Ti}, \mathrm{HA}, 3 \% \mathrm{Ag}-\mathrm{HA}$ coating and $50 \% \mathrm{Ag}$-HA coating groups were $287.0 \mathrm{~g}, 309.0 \mathrm{~g}, 324.0 \mathrm{~g}$ and $337.0 \mathrm{~g}$, respectively (Fig. 1). No significant differences in the increase in body-weight were found between the four groups at two, four and 12 weeks.

Radiological transmission imaging. The radiographs confirmed that the implants had been inserted correctly into the tibiae. There was no evidence of aseptic loosening, osteolysis or formation of heterotopic bone at two, four and 12 weeks. Histological examination. The bone formation and contact around the implant in the $3 \% \mathrm{Ag}-\mathrm{HA}$ coating group were at the same level as those in the HA coating group at 12 weeks (Fig. 2). However, inhibition of bone formation was observed in the $50 \% \mathrm{Ag}-\mathrm{HA}$ coating group. The Ti group showed plentiful bone formation around the implant, although there were gaps between the $\mathrm{Ti}$ and the bone.

Affinity indices. The mean affinity indices of bone formation in the $\mathrm{Ti}$, HA, 3\% Ag-HA and 50\% Ag-HA coating groups at two weeks were $61.0 \%, 70.4 \%, 65.7 \%$ and $29.1 \%$, respectively. The value in the $50 \% \mathrm{Ag}-\mathrm{HA}$ group was significantly different from that in the HA and $3 \% \mathrm{Ag}-$ HA groups ( $p=0.031$ and $p=0.033$, respectively). At four weeks, the mean affinity indices of bone formation in the $\mathrm{Ti}, \mathrm{HA}, 3 \% \mathrm{Ag}-\mathrm{HA}$ and $50 \% \mathrm{Ag}$-HA groups were $86.4 \%$, $73.5 \%, 74.8 \%$ and $31.7 \%$, respectively. There was a significant difference between the $\mathrm{Ti}$ and $50 \% \mathrm{Ag}$-HA groups $(\mathrm{p}=0.048)$. At 12 weeks, the indices of bone formation in the $\mathrm{Ti}, \mathrm{HA}, 3 \% \mathrm{Ag}-\mathrm{HA}$ and $50 \% \mathrm{Ag}-\mathrm{HA}$ groups were $97.3 \%, 84.9 \%, 81.0 \%$ and $40.5 \%$, respectively (Fig. 3 ). No significant differences were found between the groups $(\mathrm{p}=0.132)$. However, the $50 \%$ Ag-HA group showed less bone formation compared with the other groups. 


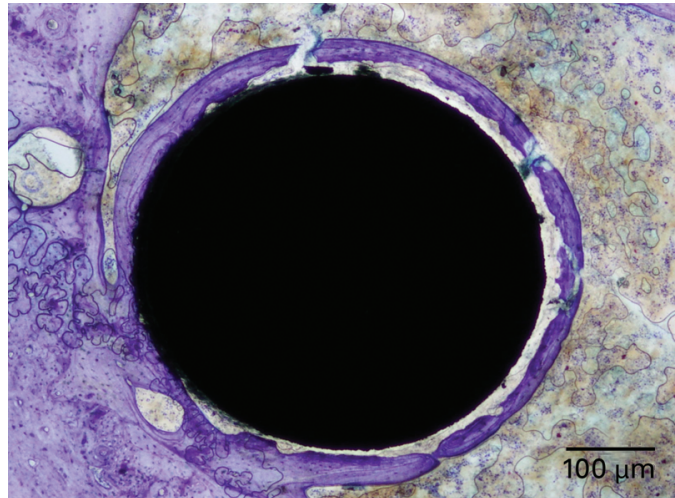

Fig. 2a

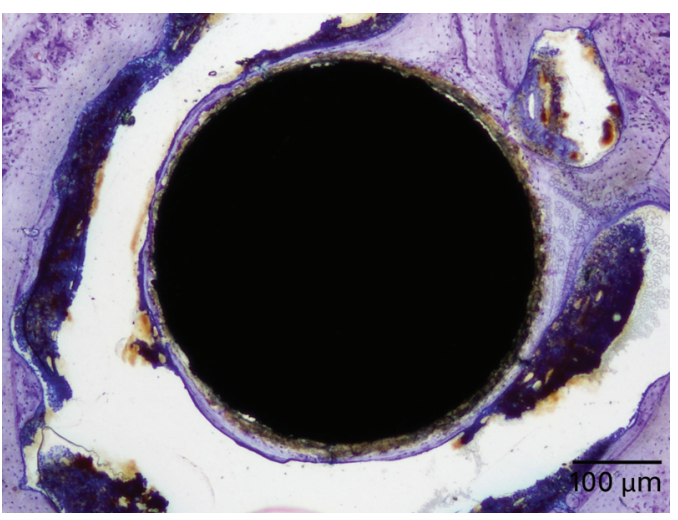

Fig. 2c

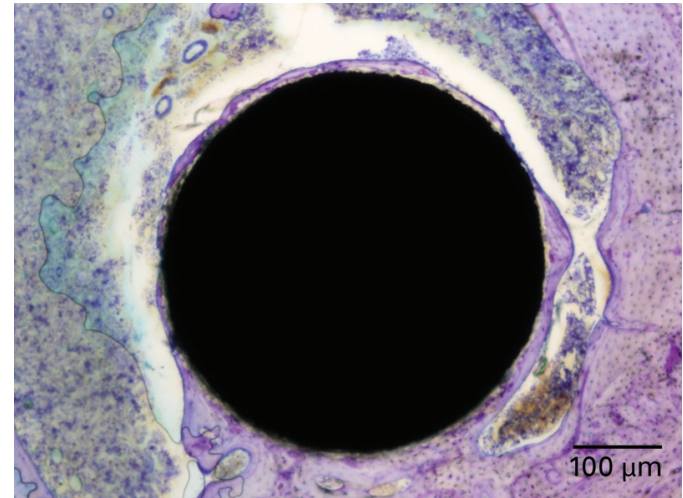

Fig. 2b

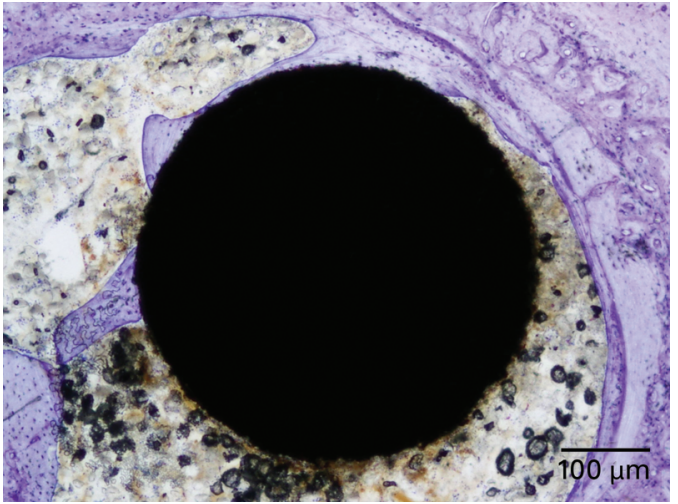

Fig. 2d

Photomicrographs of rat tibiae at 12 weeks from the a) Ti group, showing plentiful bone formation around the implant, albeit with gaps between the two, b) hydroxyapatite (HA) and c) $3 \% \mathrm{Ag}-\mathrm{HA}$ coating groups, showing good bone formation and contact around the implant, and in d) the $50 \% \mathrm{Ag}-\mathrm{HA}$ coating group, showing less bone formation around the implant compared with the other groups (toluidine blue, $\times 40$ magnification).

The mean affinity indices of bone contact in the Ti, HA, $3 \% \mathrm{Ag}-\mathrm{HA}$ and $50 \% \mathrm{Ag}-\mathrm{HA}$ groups at two weeks were $16.2 \%, 70.4 \%, 65.7 \%$ and $29.1 \%$, respectively. The values in the HA and $3 \% \mathrm{Ag}-\mathrm{HA}$ coating groups were significantly different from those in the $\mathrm{Ti}(\mathrm{p}=0.007$ and 0.006 , respectively, Tukey's HSD test) and 50\% Ag-HA ( $\mathrm{p}=0.016$ and 0.016, respectively, Tukey's HSD test) groups. At four weeks, the mean indices of bone contact in the $\mathrm{Ti}, \mathrm{HA}$, $3 \% \mathrm{Ag}-\mathrm{HA}$ and $50 \% \mathrm{Ag}-\mathrm{HA}$ groups were $31.6 \%, 63.3 \%$, $71.8 \%$ and $29.6 \%$, respectively. There were no significant differences between groups $(\mathrm{p}=0.105)$. At 12 weeks, the mean indices of bone contact in the Ti, HA, 3\% Ag-HA and $50 \%$ Ag-HA groups were $18.8 \%, 83.7 \%, 77.2 \%$ and $40.5 \%$, respectively. The values in the $\mathrm{HA}$ and $3 \% \mathrm{Ag}-\mathrm{HA}$ coating groups showed significant differences compared with those in the $\mathrm{Ti}$ ( $\mathrm{p}=0.005$ and 0.007 , respectively, Tukey's HSD test) and 50\%Ag-HA ( $\mathrm{p}=0.026$ and 0.041 , respectively, Tukey's HSD test) groups. The indices in the $3 \%$ Ag-HA group were almost the same as those in the HA group, and no significant differences were found between the 3\%Ag-HA and HA groups (ANOVA, $\mathrm{p}=0.943$ ) (Fig. 4).

Serum analysis. The mean serum concentration of $\mathrm{Ag}$ in the $\mathrm{Ti}$ and HA groups was undetectable at two, four and 12 weeks (Fig. 5). The 3\% Ag-HA and the 50\% Ag-HA groups showed a mean serum Ag concentration of 1.1 parts per billion (ppb) (0.5 to 1.3) and $5.3 \mathrm{ppb}$ (3.1 to 8.3) at two weeks respectively. The concentration of $\mathrm{Ag}$ decreased gradually over the experimental period. The mean concentration of $\mathrm{Ag}$ in the $50 \% \mathrm{Ag}-\mathrm{HA}$ group was significantly different from that in the $\mathrm{Ti}$ and HA groups at two weeks ( $p=0.042$ and 0.045 , respectively, Tukey's HSD test). Furthermore, the mean concentration in the 50\% Ag-HA group was statistically different from that in the other three groups at four ( $\mathrm{p}=0.000$ for all comparisons, Tukey's HSD test) and at 12 weeks ( $\mathrm{p}=0.046,0.025$ and 0.020 for $\mathrm{Ti}$, HA and $3 \%$ Ag-HA, respectively, Tukey's HSD test). The mean concentrations in the $3 \% \mathrm{Ag}$-HA group showed no significant difference compared with that in the Ti and HA coating groups at two, four and 12 weeks, (Ti: $\mathrm{p}=0.871$, 


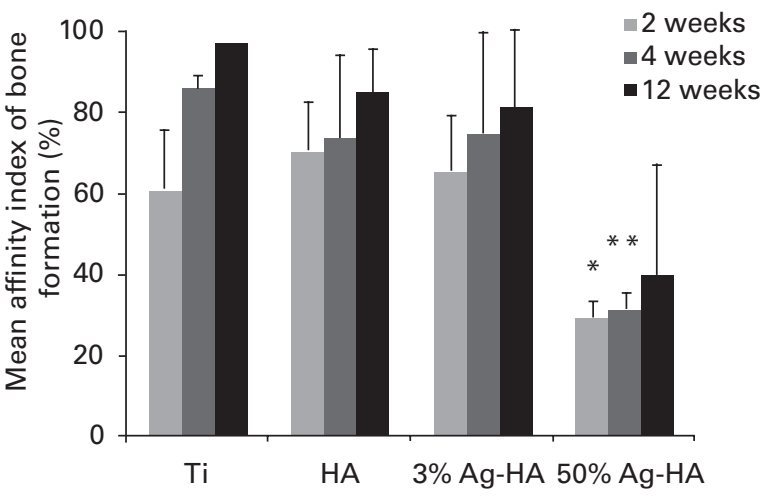

Fig. 3

Bar charts showing the mean affinity index of bone formation at two, four and 12 weeks $(*$, significant difference between the $50 \%$ Ag-hydroxyapatite (HA) coating group and both the HA and $3 \% \mathrm{Ag}-\mathrm{HA}$ coating groups at two weeks $(\mathrm{p}<0.05)$. ${ }^{*}$, significant difference between the $\mathrm{Ti}$ and the $50 \% \mathrm{Ag}-\mathrm{HA}$ coating groups at four weeks $(p<0.05))$.

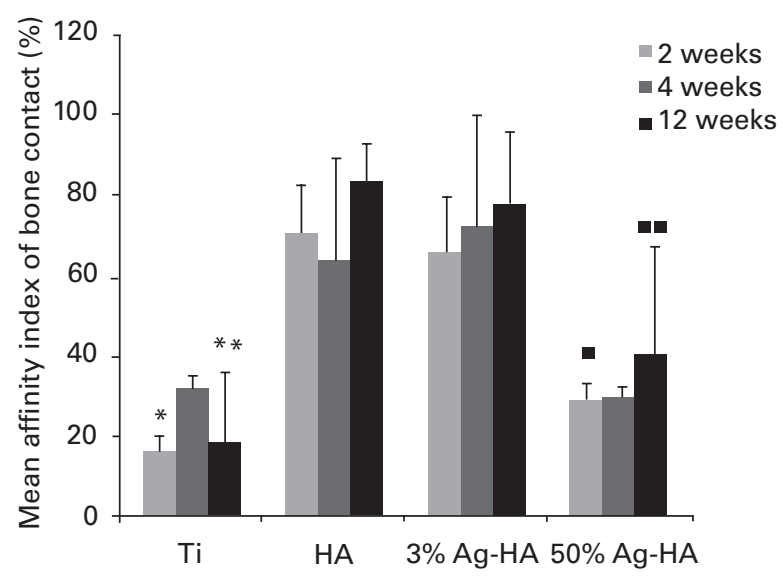

Fig. 4

Bar charts showing the mean affinity index of bone contact at two, four and 12 weeks ${ }^{*}$ and ${ }^{* *}$, significant difference between the Ti group and both the hydroxyapatite $(\mathrm{HA})$ and $3 \% \mathrm{Ag}-\mathrm{HA}$ coating groups at two and 12 weeks, respectively $(p<0.01)$. and $\square$, significant difference between the 50\%Ag-HA coating group and both the HA and $3 \% \mathrm{Ag}-\mathrm{HA}$ coating groups at two and 12 weeks, respectively $(p<0.05))$.

0.593 and 0.999 , respectively; HA: $\mathrm{p}=0.898,0.593$ and 0.999, respectively, ANOVA).

\section{Discussion}

There have been no previous studies in vivo of the osteoconductivity of an Ag-HA coating. In our study, the 3\% AgHA coating was shown to have good osteoconductivity based on the affinity indices in the rat tibia.

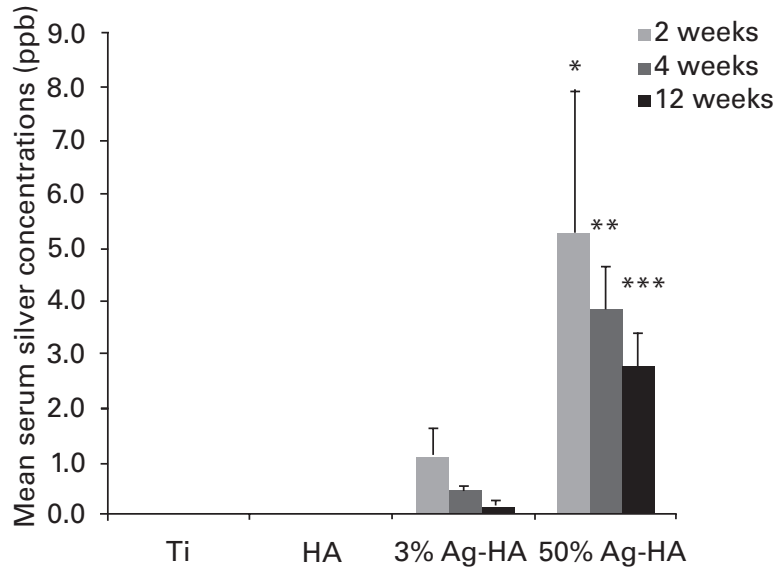

Fig. 5

Bar charts showing the mean serum Ag concentration at two, four and 12 weeks ${ }^{*}$, significant difference between the $50 \%$ Ag-hydroxyapatite (HA) coating group and the $\mathrm{Ti}$ and $\mathrm{HA}$ coating groups at two weeks $(p<0.05)$. ${ }^{* *}$ and $* * *$, significant difference between the $50 \% \mathrm{Ag}-\mathrm{HA}$ coating group and the other three groups at four weeks $(p<0.01)$ and 12 weeks $(p<0.05)$, respectively).

The affinity indices of bone formation and bone contact in the $3 \% \mathrm{Ag}-\mathrm{HA}$ coating group were the same as those in the HA group, indicating that the $3 \% \mathrm{Ag}-\mathrm{HA}$ coating may have the same osteoconductivity and strength of attachment between the bone and implant as the HA coating. Because the affinity index of bone formation has been used to evaluate osteoconductivity, the affinity indices of bone formation and bone contact were calculated in order to reveal differences in osteoconductivity for each type of coating. ${ }^{16-18}$ However, measurement of the thickness of neonatal bone and bone maturation may be necessary for more precise evaluation of osteoconductivity. ${ }^{25}$

The affinity index in the $50 \% \mathrm{Ag}$-HA coating group was lower than that in the $3 \% \mathrm{Ag}-\mathrm{HA}$ and HA groups, suggesting that the inhibition of osteoconductivity because of $\mathrm{Ag}$ toxicity depended on the dose. Alt et $\mathrm{al}^{26}$ evaluated the cytotoxicity of bone cement in vitro with the addition of nanoparticulate Ag using human osteoblasts. They found that osteoblasts formed cellular processes on the nanoparticulate $\mathrm{Ag}$ bone cement as in the non-toxic control group, indicating good biocompatibility of the cement. Hardes et $\mathrm{al}^{27}$ investigated the influence of elementary $\mathrm{Ag}$ on the behaviour of osteoblasts in vitro using human osteosarcoma cell lines, and observed a dose-dependent effect of Ag toxicity. Gosheger et $\mathrm{al}^{28}$ analysed the toxicological sideeffects of Ag-coated megaprostheses in ten rabbits, and found that the concentration of $\mathrm{Ag}$ in the blood and in the organs rose without either pathological changes in laboratory parameters or histological changes in tissues.

By contrast, it has been reported that a concentration of $\mathrm{Ag}$ in the blood of more than $300 \mathrm{ppb}$ can cause argyria, argyrosis, leukopenia and liver and kidney damage. ${ }^{29}$ Drake 
and Hazelwood ${ }^{30}$ found that the acute symptoms of overexposure to Ag nitrate include a decrease in blood pressure, diarrhoea, irritation of the stomach and decreased respiration. Chronic symptoms resulting from the intake of a low dose of Ag salts are fatty degeneration of the liver and kidneys and changes in the blood cells. ${ }^{30}$ Long-term inhalation or ingestion of soluble Ag compounds or colloidal Ag may cause argyria and/or argyrosis. ${ }^{30}$

In our study, the highest concentrations of serum $\mathrm{Ag}$ resulting from the $3 \% \mathrm{Ag}-\mathrm{HA}$ and $50 \% \mathrm{Ag}-\mathrm{HA}$ coatings were $1.1 \mathrm{ppb}$ and $5.3 \mathrm{ppb}$ at two weeks, respectively. These levels were low enough to avoid harmful effects.

Several factors may influence the reduction in the Ag concentration in the Ag-HA coating group at two, four and 12 weeks. Because the surface of the Ag-HA coating was fully covered with a thick layer of HA after soaking in simulated body fluid for 24 hours, Ag may have been absent from the surface of the coating, or its release may have been suppressed. ${ }^{21}$ Schierholz et al ${ }^{15}$ observed that in an environment containing albumin and halide ions, serum Ag ions were decreased because of absorption processes with albumin and precipitation into insoluble $\mathrm{Ag}$ chloride.

Our study evaluated the antibacterial activity of a $3 \% \mathrm{Ag}$ HA coating in vitro. ${ }^{22}$ We have shown previously that the growth of Escherichia coli, Staph. aureus and MRSA was completely suppressed on Ti discs coated with $3 \% \mathrm{Ag}-\mathrm{HA} .{ }^{22}$ Furthermore it was demonstrated that a $3 \% \mathrm{Ag}-\mathrm{HA}$ coating had in vivo antibacterial activity against MRSA, and that the concentration of $\mathrm{Ag}$ from $\mathrm{Ti}$ discs coated with 3\% Ag-HA increased to more than $50 \mathrm{ppb}$ at 48 to 72 hours. ${ }^{23}$ Silver has been shown to have antibacterial activity at concentrations as low as $35 \mathrm{ppb}$ without toxic effects to mammalian cells. ${ }^{29}$ Although the concentrations of Ag were not measured during the first two weeks after implantation in our present study, the $3 \% \mathrm{Ag}-\mathrm{HA}$ coating may have had antibacterial activities. Further studies of the $3 \% \mathrm{Ag}-\mathrm{HA}$ coating are required for the evaluation of antibacterial activity in vivo in the rat tibia and for the evaluation of the concentration of $\mathrm{Ag}$ within two weeks of implantation.

One advantage of coating with Ag-HA is that the antibacterial activity and toxicity can be arbitrarily adjusted by increasing the amount of $\mathrm{Ag}_{2} \mathrm{O}$. The Ag-HA coating can be applied to add antibacterial activity to implants without toxicity to the recipient of the implant by adjustment of the level of $\mathrm{Ag}_{2} \mathrm{O}$. By adjusting the antibacterial activity and toxicity, the Ag-HA coating may have an important role in the prevention of microbial infection after implantation.

In the rat tibia a $3 \%$ Ag-HA coating showed good osteoconductivity, and was comparable with an HA coating and a $50 \% \mathrm{Ag}$-HA coating in the inhibition of bone formation. In addition, the serum concentrations of $\mathrm{Ag}$ with an Ag-HA coating were shown to be low enough to avoid harmful effects. The 3\%Ag-HA coating had low toxicity with high osteoconductivity. However, no evidence of bactericidal activity was observed in the rat tibia. Further studies with the $3 \% \mathrm{Ag}-\mathrm{HA}$ coating are required for the evaluation of anti- bacterial activity in vivo in the rat tibia. The $3 \% \mathrm{Ag}-\mathrm{HA}$ coating on implants may be a biologically safe antibacterial biomaterial and may be of value in the reduction of surgicalsite infections related to implantation.

No benefits in any form have been received or will be received from a commercial party related directly or indirectly to the subject of this article.

\section{References}

1. National Nosocomial Infections Surveillance System. National Nosocomial Infections Surveillance (NNIS) System Report, data summary from January 1992 through June 2004, issued October 2004. Am J Infect Control 2004;32:470-85.

2. Antoci V Jr, Adams CS, Hickok NJ, Shapiro IM, Parvizi J. Vancomycin bound to Ti rods reduces periprosthetic infection: preliminary study. Clin Orthop 2007:461:8895.

3. Lucke M, Schmidmaier G, Sadoni S, et al. Gentamicin coating of metallic implants reduces implant-related osteomyelitis in rats. Bone 2003:32:521-31.

4. Alt V, Bitschnau A, Osterling J, et al. The effects of combined gentamicin-hydroxyapatite coating for cementless joint prostheses on the reduction of infection rates in a rabbit infection prophylaxis model. Biomaterials 2006;27:4627-34.

5. Stigter M, Bezemer J, de Groot K, Layrolle P. Incorporation of different antibiotics into carbonated hydroxyapatite coatings on titanium implants, release and antibiotic efficacy. J Control Release 2004;99:127-37.

6. Nable BJ, Prichard HL, Butler RD, Klitzman B, Schoenfisch MH. Inhibition of implant-associated infections via nitric oxide release. Biomaterials 2005;26:6984-90.

7. Chen W, Liu Y, Courtney HS, et al. In vitro anti-bacterial and biological properties of magnetron co-sputtered silver-containing hydroxyapatite coating. Biomaterials 2006;27:5512-17.

8. Butany J, Leask RL, Desai ND, et al. Pathologic analysis of 19 heart valves with silver-coated sewing rings. J Card Surg 2006;21:530-8

9. Logghe C, Van Ossel C, D'Hoore W, et al. Evaluation of chlorhexidine and silversulfadiazine impregnated central venous catheters for the prevention of blood stream infection in leukaemic patients: a randomized controlled trial. J Hosp Infect 1997;37:145-56.

10. Batt M, Jean-Baptiste $\mathbf{E}, \mathbf{0}$ 'Connor $\mathbf{S}$, et al. In-situ revascularisation for patients with aortic graft infection: a single centre experience with silver coated polyester grafts. Eur J Vasc Endovasc Surg 2008;36:182-8.

11. Davenport K, Keeley FX. Evidence for the use of silver-alloy-coated urethral catheters. J Hosp Infect 2005;60:298-303

12. Wang XO, Kempf M, Mott J, et al. Silver absorption on burns after the application of Acticoat: data from pediatric patients and a porcine burn model. J Burn Care Res 2009;30:341-8.

13. Hardes J, Ahrens H, Gebert C, et al. Lack of toxicological side-effects in silvercoated megaprostheses in humans. Biomaterials 2007;28:2869-75.

14. Clement JL, Harrett PS. Antibacterial silver. Met Based Drugs 1994;1:467-82.

15. Schierholz JM, Lucas LJ, Rump A, Pulverer G. Efficacy of silver-coated medical devices. J Hosp Infect 1998;40:257-62.

16. Suzuki T, Fujibayashi S, Nakagawa Y, Noda I, Nakamura T. Ability of zirconia double coated with titanium and hydroxyapatite to bond to bone under load-bearing conditions. Biomaterials 2006;27:996-1002.

17. Hara T, Hayashi K, Nakashima Y, Kanemaru T, Iwamoto Y. The effect of hydroxyapatite coating on the bonding of bone to titanium implants in the femora of ovariectomised rats. J Bone Joint Surg [Br] 1999;81-B:705-9.

18. Hayashi K, Matsuguchi N, Uenoyama K, Kanemaru T, Sugioka Y. Evaluation of metal implants coated with several types of ceramics as biomaterials. J Biomed Mater Res 1989;23:1247-59.

19. Cook SD, Thomas KA, Kay JF, Jarcho M. Hydroxyapatite-coated titanium for orthopedic implant applications. Clin Orthop 1988;232:225-43.

20. Nakashima Y, Hayashi K, Inadome T, et al. Hydroxyapatite-coating on titanium arc sprayed titanium implants. J Biomed Mater Res 1997;35:287-98.

21. Noda I, Miyaji F, Ando Y, et al. Development of novel thermal sprayed antibacterial coating and evaluation of release properties of silver ions. J Biomed Mater Res B Appl Biomater 2009;89:456-65.

22. Ando Y, Miyamoto $\mathbf{H}$, Noda I, et al. Calcium phosphate coating containing silver shows high antibacterial activity and low cytotoxicity and inhibits bacterial adhesion. Materials Science and Engineering: C 2010;30:175-80.

23. Shimazaki T, Miyamoto H, Ando Y, et al. In vivo antibacterial and silver-releasing properties of novel thermal sprayed silver-containing hydroxyapatite coating. J Biomed Mater Res B Appl Biomater 2010;92:386-9.

24. Fini M, Giavaresi G, Giardino R, Cavani F, Cadossi R. Histomorphometric and mechanical analysis of the hydroxyapatite-bone interface after electromagnetic stimulation: an experimental study in rabbits. J Bone Joint Surg [Br] 2006;88-B:123-8. 
25. No authors listed. Making method for the thin bone tissue samples for observation with optical microscope, TS T0011:2008. Technical Committee on Medical Equipment Standards Board and Japanese Industrial Standards Committee, 2008.

26. Alt V, Bechert T, Steinrücke $\mathbf{P}$, et al. An in vitro assessment of the antibacteria properties and cytotoxicity of nanoparticulate silver bone cement. Biomaterials 2004;25:4383-91

27. Hardes J, Streitburger A, Ahrens H, et al. The influence of elementary silver versus titanium on osteoblasts behaviour in vitro using human osteosarcoma cell lines. Sarcoma 2007;2007:26539.
28. Gosheger G, Hardes J, Ahrens H, et al. Silver-coated megaendoprostheses in a rabbit model: an analysis of the infection rate and toxicological side effects. Biomaterials 2004;25:5547-56.

29. Chambers CW, Proctor CM, Kabler PW. Bactericidal effect of low concentrations of silver. J Am Water Works Association 1962:206-16.

30. Drake PL, Hazelwood KJ. Exposure-related health effects of silver and silver compounds: a review. Ann Occup Hyg 2005;49:575-85. 